\title{
Evidence for a Sliding-Resistance at the Tip of the Trypanosome Flagellum
}

\author{
David Woolley, ${ }^{1 *}$ Catarina Gadelha, ${ }^{2}$ and Keith Gull ${ }^{2}$ \\ ${ }^{1}$ Department of Physiology, School of Medical Sciences, University of Bristol, \\ Bristol, United Kingdom \\ ${ }^{2}$ Sir William Dunn School of Pathology, University of Oxford, Oxford, \\ United Kingdom
}

\begin{abstract}
Motility in trypanosomes is achieved through the undulating behaviour of a single " $9+2$ " flagellum; normally the flagellar waves begin at the flagellar tip and propagate towards the base. For flagella in general, however, propagation is from base-to-tip and it is believed that bend formation, and sustained regular oscillation, depend upon a localised resistance to inter-doublet sliding - which is normally conferred by structures at the flagellar base, typically the basal body. We therefore predicted that in trypanosomes there must be a resistive structure at the flagellar tip. Electron micrographs of Crithidia deanei, Herpetomonas megaseliae, Trypanosoma brucei and Leishmania major have confirmed that such attachments are present. Thus, it can be assumed that in trypanosomes microtubule sliding at the flagellar tip is resisted sufficiently to permit bend formation. Cell Motil. Cytoskeleton 63:741-746, 2006. (c) 2006 Wiley-Liss, Inc.
\end{abstract}

Key words: flagellum; bend initiation; bend propagation; axoneme; dynein; trypanosome

\section{INTRODUCTION}

The trypanosomes are a group of kinetoplastid protozoa characterised by having only a single flagellum. In all genera, the flagellar axoneme has the standard " $9+$ 2 " ultrastructure and develops from a typical basal body located in the body of the cell [Vickerman and Preston, 1976].

The pattern of flagellar motility, however, is distinctive in that the flagellar bends are usually initiated at the tip and propagate towards the base, with the result that the cell swims with its flagellum leading [Walker and Walker, 1962; Holwill, 1965]. The trypanosome flagellum has a smooth surface: there are no mastigonemes that might complicate this interpretation of the motion [Vickerman and Preston, 1976]. In some trypanosomes, at least, the tip-to-base propagation of bends can reverse, transiently, presumably as part of an avoidingreaction [Holwill, 1965; Holwill and McGregor, 1974]; this change may be mediated through a rise in intracellular calcium concentration [Holwill and McGregor, 1975, 1976]. Bend initiation will also occur at the flagellar base after amputation of the distal flagellum [Goldstein et al.,
1970]. Thus it is usual but not mandatory for bending to be initiated at the flagellar tip.

A satisfactory theory of flagellar mechanics would allow for the possibility of bends initiating at the tip and propagating towards the base. For any flagellar bend to develop, it is thought that linkages in the flagellum must resist inter-doublet sliding [Gibbons, 1981]. Conventionally, attention has been paid to linkages located at the base of the flagellum and also to linkages distributed throughout the axoneme, such as radial spokes and nexin (or circumferential) links. Flagella amputated well beyond the basal region, when demembranated and

*Correspondence to: Dr. David M. Woolley, Department of Physiology, School of Medical Sciences, University of Bristol, University Walk, Bristol BS8 1TD, United Kingdom.

E-mail: d.m.woolley@bristol.ac.uk

Received 8 May 2006; Accepted 26 July 2006

Published online 28 September 2006 in Wiley InterScience (www. interscience.wiley. com).

DOI: $10.1002 / \mathrm{cm} .20159$ 
exposed to ATP, may show some brief irregular motion but are soon quiescent [Goldstein, 1969; Gibbons and Gibbons, 1972; Kamimura and Kamiya, 1989; Woolley and Bozkurt, 1995]. This indicates, firstly, that there are indeed distributed resistances, acting in some way to prevent telescopic sliding; and secondly, that distributed resistances alone cannot sustain rhythmic bend initiation. The emphasis is thus on the importance of a basal (or at least a localised) resistance to sliding. Some direct evidence for its importance is that when quiescent amputated axonemes are locally compressed, oscillatory bending is re-initiated [Woolley and Bozkurt, 1995]. The same result had been seen when this type of axonemal fragment had become attached at one end to a glass substrate [Douglas and Holwill, 1972]. Also, spontaneous beating can be induced by chemically cross-linking the doublets in a localised fashion [Fujimura and Okuno, 2006]. Furthermore, the bending initiated by a localised application of ATP was explained in terms of localised resistances [Shingyogi et al., 1977]. Finally, in theoretical studies of bend formation, a basal resistance has always seemed to be a necessary postulate e.g. [Lindemann, 1994]. The experimental studies (above), together with comparative morphological work, strongly indicate that there is no specific need, within a basal resistance, for a basal body [further references in Woolley and Bozkurt, 1995]. The basal resistance may nevertheless permit some small degree of shear displacement - and basal elastic deformation may be important in sustaining some types of oscillation [Vernon and Woolley, 2004].

We have considered the hypothesis that "bend formation requires a localised resistance beyond the site from which the bend will propagate". From this we predicted that linkages between the doublets would be found at the flagella tip in the trypanosomes. We have tested this prediction using electron microscopy.

\section{MATERIALS AND METHODS}

The trypanosomes studied, representing four of the Trypanosomatidae family, were Crithidia deanei, Herpetomonas megaseliae, Trypanosoma brucei and Leishma- nia major. Cells from the first three species named were harvested from culture, washed in phosphate-buffered saline and fixed as pellets in $2.5 \%$ glutaraldehyde in $0.1 \mathrm{M}$ phosphate buffer ( $\mathrm{pH} 7.0$ ) for $1 \mathrm{~h}$ at room temperature, postfixed in $0.5 \%$ osmium tetroxide in the same buffer for $30 \mathrm{~min}$ at $4{ }^{\circ} \mathrm{C}$, dehydrated, embedded in Epon 812, sectioned and stained conventionally. Cells from Leishmania major were prepared as whole-mounts. They were allowed to settle onto carbon coated, charged Formvar grids. Lipid extraction was achieved using $1 \%$ (vol/vol) NP-40 in PEME buffer (100 mM PIPES, $1 \mathrm{mM}$ $\mathrm{MgSO}_{4}, 0.1 \mathrm{mM}$ EDTA, $2 \mathrm{mM}$ EGTA; then the cells were fixed in $2.5 \%$ glutaraldehyde in PEME buffer and negatively stained with $0.7 \%$ gold thioglucose in water.

\section{RESULTS}

Longitudinal, sagittal sections through the flagellar tip of $C$. deanei showed that the axoneme ends bluntly, with no diminution of structure. Two apparently annular densities were seen, one associated with the doublet microtubules and one with the central singlets (Fig. 1). Serial transverse sections confirmed the annular morphology and showed that the peripheral annulus was related to the deep surface of the doublets (Fig. 2). Sometimes these annuli were seen to be tilted (Fig. 3).

Similar thin sections of $H$. megaseliae also showed electron dense material in an equivalent position, having a more disc-like appearance and also sometimes appearing to be tilted (Figs. 4 and 5). A few longitudinal sections of flagellar tips were obtained for T. brucei. Again, dense material was found at the microtubule terminations, presumably as annular linkages between them (Fig. 6).

We considered the possibility that the linkages described might not be a feature of mature flagella but might instead be associated with the assembly of the axoneme in growing flagella. To resolve this question we examined negatively-stained preparations of Leishmania major that contained dividing cells, each bearing an old, mature flagellum and a new one that had not yet reached its full length (Fig. 7). At higher magnification,
Figures 1-6. Fig. 1. The flagellar tip of Crithidia deanei in longitudinal section. There is electron dense material at the level of the termination of the central pair (arrowhead) and also at the level of the termination of the doublet microtubules (arrow). Scale bar $0.2 \mu \mathrm{m}$ applies also to Fig. 2. Fig. 2. a-d. The flagellar tip of C.deanei in serial transverse sections (separation $\sim 60 \mathrm{~nm}$ ). Fig. 2a shows the standard $9+2$ morphology; $2 \mathrm{~b}$ and $2 \mathrm{c}$ show the annular density at the level of the termination of the central pair; and $2 \mathrm{~d}$ shows circumferential linkages making up an annulus at the level of the doublet terminations, on the deep side of each doublet. Fig. 3. Another flagellar tip from C.deanei showing tilting of the annulus around the doublet termina- tions (only partially contrasted). Scale bar $0.2 \mu \mathrm{m}$. Fig. 4 . The flagellar tip of Herpetomonas megaseliae in longitudinal section. The terminal densities generally conform to those seen in $C$. deanei. This example shows tilting. Scale bar $0.2 \mu \mathrm{m}$. Fig. 5. Another flagellar tip from $H$. megaseliae, with a lower degree of tilting. This image includes material of the paraflagellar rod (arrowed). Scale bar $0.2 \mu \mathrm{m}$. Fig. 6. The flagellar tip of Trypanosoma brucei in longitudinal section. Electron dense material occurs at the terminations of the central pair and also of the doublet microtubules. It is inferred that these are also annular linkages. Some material of the paraflagellar rod is included (arrowed). Scale bar $0.2 \mu \mathrm{m}$. 

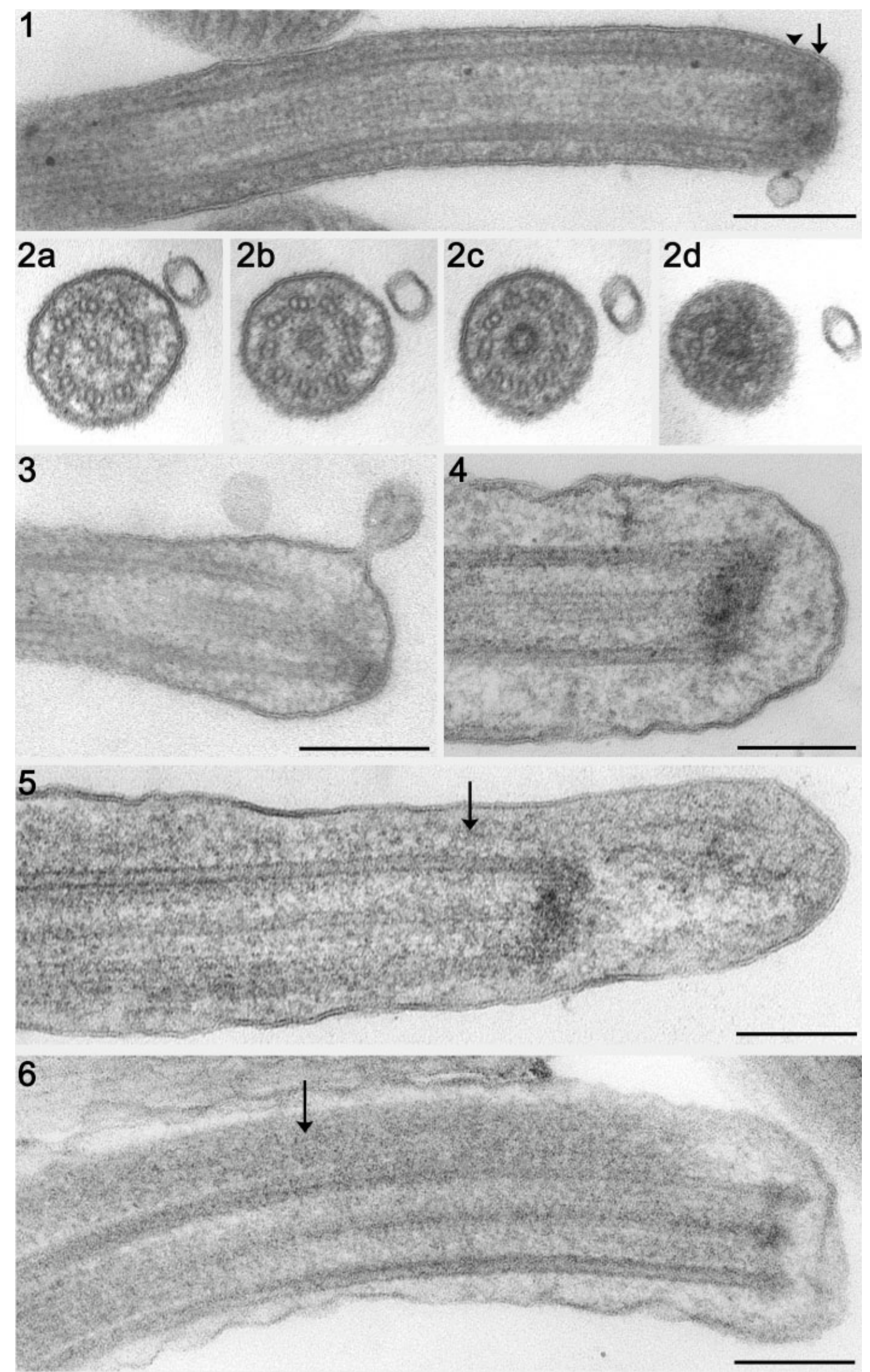

Figures 1-6. 

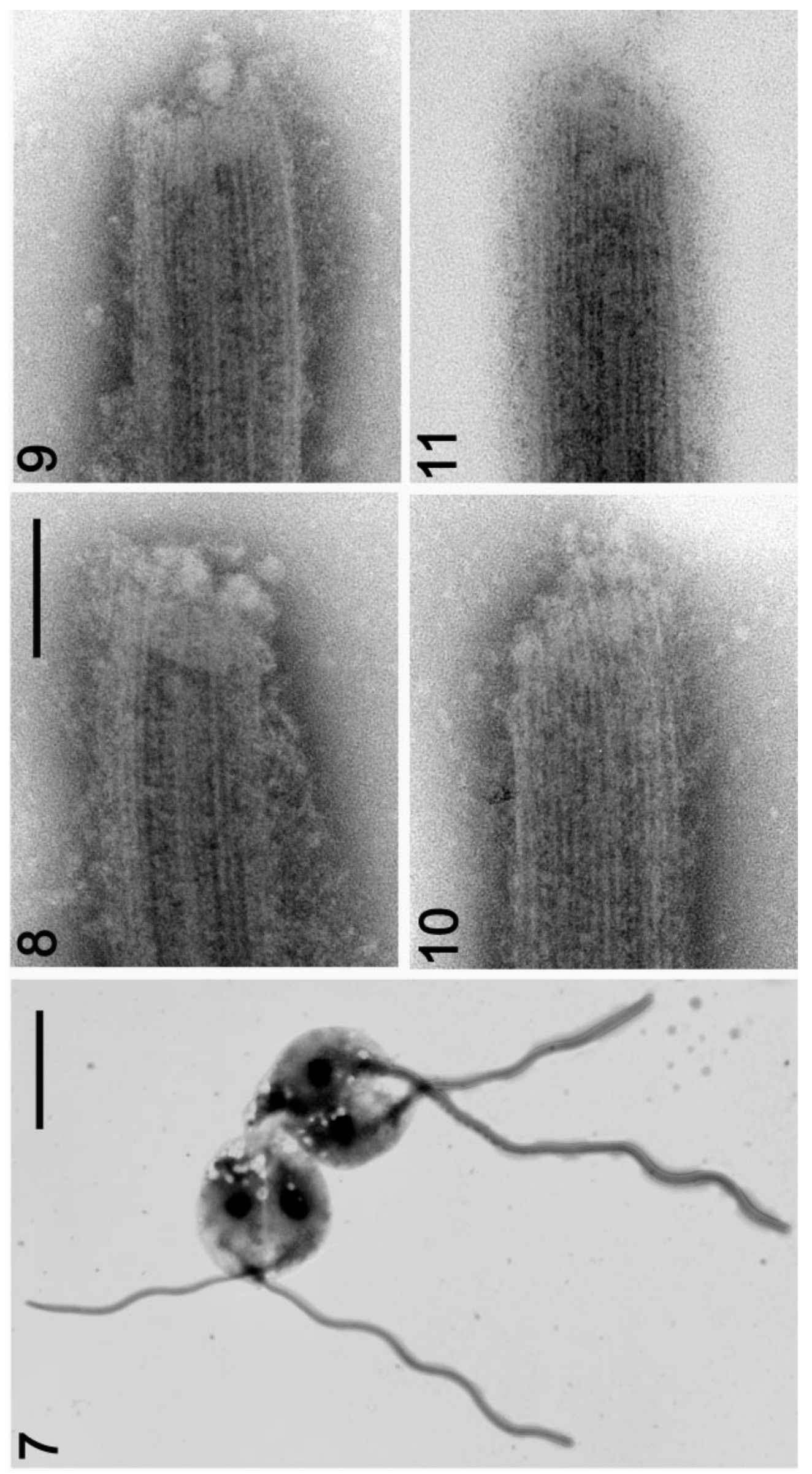

के 롤

6.0.

揱自

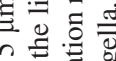

호원

을

记

के

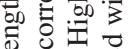

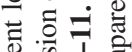

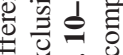

政

현

응 च

응 흐 है

क

额 $\approx$ 熍

옹

궁

证

ㅎํ월

주

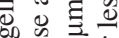

$\rightleftarrows$ 둥

3

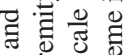

등 장

\& 든

的安

긍

on bo

당

次

吾吾

60

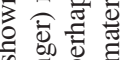

흘

응

으은

害苛

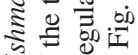

ड़

능. 을

.ํㅡㄹ

要

은 要

竞

일

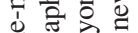

की

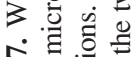

응.

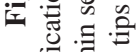

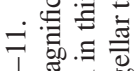

굘

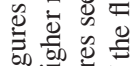

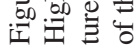


the existence of linking structures at the tip could be established by the exclusion of negative stain from the region between the doublets and around the central pair of microtubules. The old flagella always showed well organised material in these regions (Figs. 8 and 9) whereas in the new, growing flagella, such material was either apparently absent or present in smaller amounts and less well defined (Figs. 10 and 11). This conclusion is based on a comparison of growing and mature flagella from 7 cells, together with 6 additional micrographs showing the tips of mature flagella. We conclude that the linkages are characteristic of mature flagella and cannot be explained as features of flagellar assembly

\section{DISCUSSION}

The morphological features described are consistent with there being a resistance to sliding at the tip of the mature flagellum in trypanosomes. It remains to be established at what stage in flagellogenesis these features become effective. For the tip to act as an effective anchorage, one would expect it to be blunt-ended and to have electron-dense connections between the doubtlets. One might also expect evidence of some shear strain (tilting) in these structures, as we have seen.

In contrast, in most ciliary and flagellar axonemes, where bends propagate from base-to-tip, the distal end of the axoneme is tapered and has been shown to permit sliding displacements [Satir, 1968; Sale and Satir, 1976; Woolley and Nickels, 1985; Vernon and Woolley, 2002].

However, in cilia that propel mucus, tip anchorages certainly exist [Dentler and LeCluse, 1982] and such cilia presumably initiate their bends at the base. Therefore tip linkages per se do not make tip-initiation obligatory, as is evident also from the fact that the trypanosome Crithidia can initiate new bends at the flagellar base during the transient avoiding reaction described by Holwill [1965]. Our view is that the linkages are a sliding resistance and as such they enable rhythmic tip initiation to be within the repertoire of motion. This view is consistent with experimental work involving the imposition of resistances to sliding [Woolley and Bozkurt, 1995; Fujimura and Okuno, 2006]. We are assuming, perhaps naively, that the direction of propagation is a mechanical consequence of the extremity at which a bend is formed.

Apart from the trypanosomes, there are other types of flagella that can propagate bends from tip-to-base in a reversible manner. In one of these, the Myzostomum sperm flagellum, there is good evidence for a tip anchorage [Afzelius, 1983]. Evidence from Turritella spermatozoa is inconclusive [Afzelius and Dallai, 1983]. For the remaining examples, no descriptions of the tip have yet been given [Baccetti et al., 1989; Curtis et al., 1989; Curtis and Benner, 1991].

It is not easy to apply the conventional sliding doublet theory to a flagellum that has sliding-resistances at both its proximal and distal ends. For a flagellum having planar undulations, the 'packet' of sliding associated with a bend approaching the tip (or the base, as in trypanosomes) must be accommodated by torsion if sufficient sliding cannot occur at the tip (or base) [Dentler and LeCluse, 1982]. This is likely to result in a departure from planarity of the entire wave. For Crithidia, where much of the flagellum projects away from the cell body, the wave is "predominantly planar', though the organism rotates about its axis of progression [Holwill, 1965]. It is likely that the axis of sliding is enforced by the attachments between doublets 4-7 and the paraflagellar rod that is typical of trypanosomes, including Crithidia [reviewed by Gull, 1999; Gadelha et al., 2005]. Hirons and Holwill [1986] reported that for base-to-tip propagation in Crithidia bends approaching the tip produced a turning action on the whole organism but it is not clear how consistently this happened. It is quite possible that the rotation of the organism is due to the shape of the cell body, and due also, in Trypanosoma spp. to the helical line-of-attachment of the flagellum to the cell body [Hill, 2003].

The presence of the flagellar tip anchorage in the trypanosomes may explain one feature of the investigations into the role of $\mathrm{Ca}^{2+}$ ions in reversing the wave direction. In those experiments [Holwill and McGregor, 1976], it seems that the persistence of tip-to-base propagation, at low calcium concentration, depended on the severity of the extraction protocol. The persistence was greatest after mechanical separation, less after glycerolation and least after detergent-extraction. This could be explained by the progressive breakdown of the anchoring structures of the tip.

It may be worth explaining that reversing the direction of flagellar bend propagation does not imply a reversal of the polarity of force generation by the dynein arrays. For a planar bend originating at the basal end, the active set of dyneins on doubtlet $n$ will cause doublet $n+1$ to slide towards the tip [Sale and Satir, 1977]. Let us say this produces a bend in the +ve direction. Now, imagine the same doublet pair involved in generating a bend at the tip end. Doublet $n+1$ cannot slide towards the tip because of the tip anchorage. Therefore force generation by the dyneins on doubtlet $n$ will cause doublet $n$ to move towards the base and a bend will form in the -ve direction. The direction of force generation is unchanged but the resulting bend takes the opposite direction. However, reverse propagation of bends does mean that any necessary interaction between adjacent dynein arms can occur in either the proximo-distal or the disto-proximal direction. 
In conclusion, our results sustain the hypothesis that bend initiation on a flagellum requires a sliding resistance located beyond the site from which the formed bend will propagate. Normally such resistances will be permanent and either basal or - in rare instances such as the trypanosomes - both basal and terminal. However, in unusual circumstances the necessary resistance can be functional, as when dynein cross-bridges seem to be effective resistances [Woolley and Vernon, 2002], or when the proximal flagellum is physically constrained from bending/sliding by attachment to glass [Brokaw, 1982; Woolley, 2005].

\section{ACKNOWLEDGMENTS}

D.M.W. acknowledges the support of the BBSRC (UK) and the expert technical help of Gini Tilly. Thanks also to Debbie Carter for preparing the illustrations and to Lisa Nicholas and Pat East for preparing the text. C. G. and K. G. thank the Wellcome Trust and the Human Frontiers Science Program for support.

\section{REFERENCES}

Afzelius BA. 1983. The spermatozoon of Myzostomum cirriferum (Annelida, Myzostomida). J Ultrastruct Res 83:58-68.

Afzelius BA, Dallai R. 1983. The paired spermatozoa of the marine snail Turritella communis Lamarck (Mollusca, Mesogastropoda). J Ultrastruct Res 85:311-319.

Baccetti B, Gibbons BH, Gibbons IR. 1989. Bidirectional swimming in spermatozoa of Tephritid flies. J Submicrosc Cytol Pathol 21:619-625.

Brokaw CJ. 1982. Models for oscillation and bend propagation by flagella. Symp Soc Exp Biol 35:313-338.

Curtis SK, Benner DB. 1991. Movement of spermatozoa of Megaselia scalaris (Diptera: Brachycera: Cyclorrhapha: Phoridae) in artificial and natural fluids. J Morphol 210:85-99.

Curtis SK, Benner DB, Musil G. 1989. Ultrastructure of the spermatozoon of Megaselia scalaris Loew (Diptera: Brachycera: Cyclorrhapha: Phoridea: Phoridae). J Morphol 200:47-61.

Dentler WL, LeCluse EL. 1982. Microtubule capping structures at the tips of tracheal cilia: Evidence for their firm attachment during ciliary bend formation and the restriction of microtubule sliding. Cell Motil 2:549-572.

Douglas GJ, Holwill MEJ. 1972. Behaviour of flagella isolated from Crithidia oncopelti. J Mechanochem Cell Motil 1:213-223.

Fujimura M, Okuno M. 2006. Requirement of the fixed end for spontaneous beating in flagella. J Exp Biol 209:1336-1343.

Gadelha C, Wickstead B, de Souza W, Gull K, Cunha-e-Silva N. 2005. Cryptic paraflagellar rod in endosymbiont-containing Kinetoplastid protozoa. Eukaryot Cell 4:516-525.

Gibbons IR. 1981. Cilia and flagella of eukaryotes. J Cell Biol 91:107S-124S.
Gibbons BH, Gibbons IR. 1972. Flagellar movement and adenosine triphosphatase activity in sea urchin sperm extracted with Triton X-100. J Cell Biol 54:79-97.

Goldstein SF. 1969. Irradiation of sperm tails by laser microbeam. J Exp Biol 51:431-441.

Goldstein SF, Holwill MEJ, Silvester NR. 1970. The effects of laser microbeam irradiation on the flagellum of Crithidia (Strigomonas) oncopelti. J Exp Biol 53:401-409.

Gull K. 1999. The cytoskeleton of trypanosomatid parasites. Annu Rev Microbiol 53:629-655.

Hill KL. 2003. Biology and mechanism of trypanosome cell motility. Eukaryot Cell 2:200-208.

Hirons MR, Holwill MEJ. 1986. Patterns of flagellar wave propagation in Crithidia oncopelti at increased viscosities. Cell Motil Cytoskeleton 6:99-104.

Holwill MEJ. 1965. The motion of Strigomonas oncopelti. J Exp Biol 42:125-137.

Holwill MEJ, McGregor JL. 1974. Micromanipulation of the flagellum of Crithidia oncopelti. J Exp Biol 60:437-444.

Holwill MEJ, McGregor JL. 1975. Control of flagellar wave movement in Crithidia oncopelti. Nature 255:157, 158.

Holwill MEJ, McGregor JL. 1976. Effects of calcium on flagellar movement in the trypanosome Crithidia oncopelti. J Exp Biol 65:229-242.

Kamimura S, Kamiya R. 1989. High-frequency, nanometre-scale vibration in 'quiescent' flagellar axonemes. Nature 340:476-478.

Lindemann CB. 1994. A "geometric clutch" hypothesis to explain oscillations of the axoneme of cilia and flagella. J Theor Biol 168:175-189.

Sale WS, Satir P. 1976. Splayed Tetrahymena cilia. J Cell Biol 71:589-605.

Sale WS, Satir P. 1977. Direction of active sliding of microtubules in Tetrahymena cilia. Proc Natl Acad Sci USA 74:2045-2049.

Satir P. 1968. Studies on cilia III. Further studies on the cilium tip and a "sliding filament" model of ciliary motility. J Cell Biol 39: 77-94.

Shingyoji C, Murakami A, Takahashi K. 1977. Local reactivation of Triton-extracted flagella by iontophoretic application of ATP. Nature 265:269, 270.

Vernon GG, Woolley DM. 2002. Microtubule displacements at the tips of living flagella. Cell Motil Cytoskeleton 52:151-160.

Vernon GG, Woolley DM. 2004. Basal sliding and the mechanics of oscillation in a mammalian sperm flagellum. Biophys $\mathrm{J} 87$ : 3934-3944.

Vickerman K, Preston TM. 1976. Comparative cell biology of the Kinetoplastid flagellates. In: Lumsden WHR, Evans DA, editors. Biology of the Kinetoplastida, Vol. 1. London: Academic Press. pp 35-130.

Walker PJ, Walker JC. 1962. Movement of trypanosome flagella. J Protozool 10 (Suppl):109.

Woolley DM. 2005. A flagellar oscillation driven by mechanical feedback. Mol Biol Cell 16 (Suppl):654A.

Woolley DM, Bozkurt HH. 1995. The distal sperm flagellum: its potential for motility after separation from the basal structures. J Exp Biol 198:1469-1481.

Woolley DM, Nickels SN. 1985. Microtubule termination patterns in mammalian sperm flagella. J Ultrastruct Res 90:221-234.

Woolley DM, Vernon GG. 2002. Functional state of the axonemal dyneins during flagellar bend propagation. Biophys J 83:2162-2169. 